\title{
Parameters estimation based on progressively censored data from inverse Weibull distribution
}

\author{
Mostafa M. MohieEl-Din ${ }^{1}$, Fathy H. Riad ${ }^{2}$, Mohamed A. El-Sayed ${ }^{3,4}$ \\ ${ }^{1}$ Dept. of Mathematics, Faculty of Science, Al-Azhar University, Egypt \\ ${ }^{2}$ Dept. of Mathematics, Faculty of Science, Minia University, Egypt \\ ${ }^{3}$ Dept. of Mathematics, Faculty of Science in Qena, South Valley University, Egypt \\ ${ }^{4}$ Dept of CS, CIT College, Taif University, KSA
}

\section{Email address:}

fathyhamdi2008@yahoo.com(F. H. Riad),drmasayed@yahoo.com(M. A. El-Sayed)

\section{To cite this article:}

Mostafa M. MohieEl-Din, Fathy H. Riad, Mohamed A. El-Sayed. Parameters Estimation Based on Progressively Censored Data from Inverse Weibull Distribution. American Journal of Theoretical and Applied Statistics. Vol. 2, No. 6, 2013, pp. 149-153.

doi: 10.11648/j.ajtas.20130206.11

\begin{abstract}
In this article, our main aim is to investigate the parameters estimation of inverse Weibull distribution in the frame work of progressively type II. We consider the censored sample from a two parameters inverse Weibull. The point estimators of the parameters derived by using the maximum likelihood method. The exact joint confidence region and confidence interval for the parameters are obtained. A numerical example is provided to illustrate the proposed. estimation methods developed here.
\end{abstract}

Keywords: Joint Confidence Region, Maximum Likelihood Estimator, Progressively Type II Censored Sample, Confidence Interval

\section{Introduction}

Censoring is very common in life tests. It usually applies when exact lifetimes are known for only a portion of the products and the remainder of the lifetimes are known only to exceed certain values under a life test. In the study, we consider a censoring scheme called progressive type II censoring. Under this scheme, $\mathrm{n}$ units are placed on a test at time zero, with $\mathrm{m}$ failures to be observed. When the first failure is observed, $r_{1}$ of the surviving units are randomly selected and removed. At the second observed failure, $r_{2}$ of the surviving units are randomly selected and removed. This experiment stops at the time when the $m$-th failure is observed and the remaining $r_{m}=n-r_{1}-r_{2}-\ldots-r_{m-1}-m$ surviving units are all removed. The statistical inference on the parameters of some distributions under progressive type-II censoring has been investigated by several authors such as $[1,2,3,4,5]$. The Inverse Weibull distribution plays an important role in many applications, including the dynamic components of diesel engine and several data set such as the times to breakdown of an insulating fluid subject to the action of a constant tension, see [6]. Calabria and Pulcinia in [7] provided an interpretation of the inverse Weibull distribution in the context of the load strength relationship for a component. Maswedah, [8] has fitted the inverse Weibull distribution to the flood data reported in [9]. For more details on the inverse Weibull distribution, see, for example Johnson et al. [10], Murthy et al. [11] and Mohie El-Din et al [12] .

The inverse Weibull model was developed by Erto [13]. The probability density function $(p d f)$ of the random variable $X$ having a three-parameter inverse Weibull distribution with location parameter $\alpha \geq 0$, scale parameter $\eta>0$ and shape parameter $\beta>0$ is given by Erto [13], and Maru et al. [14]. The probability density function ( $p d f)$ of a two parameter inverse Weibull distribution has the form:

$$
f(x ; \alpha, \beta, \eta)=\left\{\begin{array}{cc}
\frac{\beta}{\eta}\left(\frac{\eta}{x-\alpha}\right)^{\beta+1} e^{-\left(\frac{\eta}{x-\alpha}\right)^{\beta}}, & x>\alpha, \quad \eta, \beta>0, \\
0, & x \leq \alpha,
\end{array}\right.
$$

If $\alpha=0$, the resulting distribution is called the two-parameter inverse Weibull distribution.

In this article, we consider progressively type II censored data from a two parameters inverse Weibull distribution. We obtain the maximum likelihood estimators of the parameters in Section 2. In Section 3, we derive an exact confidence interval for the parameter $\beta$ and an exact joint confidence region for the parameters $\beta$ and $\eta$. A numerical example 
is presented for illustration in Section 4.

\section{Point Estimations of Parameters}

In this section, the maximum likelihood estimators (MLEs) for the parameters of the inverse Weibull distribution based on progressive type II censoring are derived. The cumulative distribution ( $c d f)$ of the inverse Weibull distribution as follows:

$$
F(x ; \alpha, \beta, \eta)=e^{-\left(\frac{\eta}{x-\alpha}\right)^{\beta}}, x>\alpha, \eta, \beta>0 .
$$

Let $\quad X_{1: m: n}, X_{2: m: n}, \cdots, X_{m: m: n} \quad$ be $\quad$ a progressively type II censored sample from a three parameter inverse Weibull distribution, with censoring scheme $r=\left(r_{1}, \cdot, \cdot, \cdot, r_{m}\right)$. The likelihood function is given by:

$$
\begin{array}{r}
L(\beta, \alpha, \eta)=k \prod_{i=1}^{m} f\left(x_{i: m: n}\right)\left[1-F\left(x_{i: m: n}\right)\right]^{i} \\
=\frac{k \beta^{m}}{\eta^{m}} \prod_{i=1}^{m}\left[\frac{x_{i}-\alpha}{\eta}\right]^{-(\beta+1)} e^{-\left[\frac{x_{i}-\alpha}{\eta}\right]^{\beta}}\left[1-e^{-\left[\frac{x_{i}-\alpha}{\eta}\right]^{\beta}}\right]^{r_{i}}
\end{array}
$$

where $k=n\left(n-1-r_{1}\right)\left(n-2-r_{1}-r_{2}\right) \cdots\left(n-m+1-r_{1}-\cdots-r_{m-1}\right)$.

For simplicity of notation, we will use $x_{i}$ instead of $x_{i: m: n}$. The log-likelihood function may then be written as:

$$
\begin{aligned}
£= & \log L(\beta, \alpha, \eta)=\log k+m \log \beta \\
& +m \beta \log (\eta)-(1+\beta) \sum_{i=1}^{m} \log \left(x_{i}-\alpha\right) \\
& -\eta^{\beta} \sum_{i=1}^{m}\left(x_{i}-\alpha\right)^{-\beta}+\sum_{i=1}^{m} r_{i} \log \left[1-e^{-\left[\frac{x_{i}-\alpha}{\eta}\right]^{\beta}}\right]
\end{aligned}
$$

We obtain the estimators of $\beta$ and $\eta$ of by differentiating (4) with respect to $\beta$ and $\eta$ respectively and equating to zero, in this case we have :

$$
\begin{aligned}
& \frac{\partial £}{\partial \eta}=m-\sum_{i=1}^{m}\left(\frac{x_{i}-\alpha}{\eta}\right)^{-\beta} \\
& +\sum_{i=1}^{m} \frac{r_{i}\left(\frac{x_{i}-\alpha}{\eta}\right)^{-\beta} e^{-\left(\frac{x_{i}-\alpha}{\eta}\right)^{-\beta}}}{1-e^{-\left(\frac{x_{i}-\alpha}{\eta}\right)^{-\beta}}}=0,
\end{aligned}
$$

and

$$
\begin{aligned}
& \frac{\partial £}{\partial \beta}=\frac{m}{\beta}+m \log \eta \\
& +\sum_{i=1}^{m}\left[\left(\frac{x_{i}-\alpha}{\eta}\right)^{-\beta} \log \left(\frac{x_{i}-\alpha}{\eta}\right)\right] \\
& -\sum_{i=1}^{m}\left[\frac{r_{i} e^{-\left(\frac{x_{i}-\alpha}{\eta}\right)^{-\beta}} \log \left(\frac{x_{i}-\alpha}{\eta}\right)}{1-e^{-\left(\frac{x_{i}-\alpha}{\eta}\right)^{-\beta}}}\right]=0 .
\end{aligned}
$$

The MLEs $\hat{\eta}$ and $\hat{\beta}$ can be obtained by:

$$
\begin{aligned}
& m+\sum_{i=1}^{m}\left(\frac{x_{i}-\hat{\alpha}}{\hat{\eta}}\right)^{-\hat{\beta}}+ \\
& \frac{\sum_{i=1}^{m} r_{i}\left(\frac{x_{i}-\hat{\alpha}}{\hat{\eta}}\right)^{-\hat{\beta}} e^{-\left(\frac{x_{i}-\hat{\alpha}}{\hat{\eta}}\right)^{-\hat{\beta}}}}{1-e^{-\left(\frac{x_{i}-\hat{\alpha}}{\hat{\eta}}\right)^{-\hat{\beta}}}}=0 .
\end{aligned}
$$

and

$$
\begin{aligned}
& \frac{m}{\hat{\beta}}+m \log \hat{\eta} \\
& +\sum_{i=1}^{m}\left[\left(\frac{x_{i}-\hat{\alpha}}{\hat{\eta}}\right)^{-\hat{\beta}} \log \left(\frac{x_{i}-\hat{\alpha}}{\hat{\eta}}\right)\right] \\
& -\sum_{i=1}^{m}\left[\frac{r_{i} e^{-\left(\frac{x_{i}-\hat{\alpha}}{\hat{\eta}}\right)^{-\beta}} \log \left(\frac{x_{i}-\hat{\alpha}}{\eta}\right)}{\left.1-e^{-\left(\frac{x_{i}-\alpha}{\eta}\right)^{-\beta}}\right]=0 .}\right.
\end{aligned}
$$

since it can solve the equations (7) and (8) for $\hat{\beta}$ and $\hat{\eta}$ by numerical solution.

\section{Interval Estimations of Parameters}

In this section, an exact confidence interval for $\beta$ and $\eta$ an exact joint confidence region for $\beta$ and $\eta$ are investigated. Let $X_{1}<X_{2}<\cdots<X_{m-1}<X_{m}$ denote a progressively type II censored sample from a three parameter inverse Weibull distribution, with censoring scheme $\quad r=\left(r_{1}, \cdots, r_{m}\right) \quad$. Further, let $Z_{i}=\left(\frac{X_{i}-\alpha}{\eta}\right)^{\beta}, i=1, \cdots, m$. It can be seen that $Z_{1}<Z_{2}<\cdots<Z_{m-1}<Z_{m}$ is a progressively type II censored sample from an exponential distribution with mean 1 . Let us consider the following transformation: 


$$
\left\{\begin{array}{l}
S_{1}=n Z_{1} \\
S_{2}\left(n-r_{1}-1\right)\left(Z_{2}-Z_{1}\right) \\
\cdot \\
S_{m}=\left(n-r_{1}-\cdots-r_{m-1}-m+1\right)\left(Z_{m}-Z_{m-1}\right) .
\end{array}\right.
$$

Thomas and Wilson [15] proved that the generalized spacing $S_{1}, S_{2}, \cdots, S_{m}$ as defined in (9), are independent and identically distributed as an exponential distribution with mean 1 . Hence,

$$
V=2 S_{1}=2 n Z_{1}
$$

has a chi-square distribution with 2 degrees of freedom and

$$
U=2 \sum_{i=2}^{m}=2\left[\sum_{i=1}^{m}\left(r_{i}+1\right) Z_{i}-n Z_{1}\right]
$$

has a chi-square distribution with $2 m-2$ degrees of freedom. We can also find that $U$ and $V$ are independent random variables. Let

$$
T_{1}=\frac{U}{(m-1) V}=\frac{\sum_{i=1}^{m}\left(r_{i}+1\right) Z_{i}-n Z_{1}}{n(m-1) Z_{1}},
$$

and

$$
\begin{aligned}
T_{2} & =U+V=2 \sum_{i=1}^{m}\left(r_{i}+1\right) Z_{i} \\
& =2 \eta^{\beta} \sum_{i=1}^{m}\left(r_{i}+1\right)\left(x_{i}-\alpha\right)^{\beta} .
\end{aligned}
$$

It is easy to show that $T_{1}$ has an $F$ distribution with $2 m-2$ and 2 degrees of freedom and $T_{2}$ has a chi-square distribution with $2 m$ degrees of freedom.

Furthermore, by Johnson et al. [10], $T_{1}$ and $T_{2}$ are independent.

To obtain the confidence interval for $\beta$ and the joint confidence region for $\beta$ and $\eta$, we use the following lemma.

\section{Lemma}

Suppose that $w_{1}<w_{2}<\cdots<w_{m}, w>\alpha$. Let

$T_{1}(\beta)=\frac{\sum_{i=1}^{m}\left(r_{i}+1\right)\left(w_{i}-\alpha\right)^{\beta}-n\left(w_{1}-\alpha\right)^{\beta}}{n(m-1)\left(w_{1}-\alpha\right)^{\beta}}$ Then,

$T_{1}(\beta)$ is strictly increasing in $\beta$ for any $\beta>0$. Furthermore, if $t>0$, the equation $T_{1}(\beta)=t$ has unique solution for any $\beta>0$.

\section{Theorem 1.}

Suppose that $X_{i}, i=1, \cdots, m$, are the order statistics of a progressively type-II censored sample of size $n$ from a three parameter inverse Weibull distribution, with censoring scheme $\left(r_{1}, \cdots, r_{m}\right) \quad$. Then a $100(1-\phi) \%$ confidence interval for $\beta$ is:

$$
\begin{aligned}
\Psi\left(X_{1}, \cdots, X_{m},\right. & \left.F_{\left(1-\frac{\phi}{2}\right)(2 m-2,2)}\right)<\beta \\
& <\Psi\left(X_{1}, \cdots, X_{m}, F_{\left(\frac{\phi}{2}\right)(2 m-2,2)}\right) .
\end{aligned}
$$

Proof. From (10), we obtain

$$
\begin{aligned}
T_{1} & =\frac{\sum_{i=1}^{m}\left(r_{i}+1\right) Z_{i}-n Z_{1}}{n(m-1) Z_{1}} \\
& =\frac{\sum_{i=1}^{m}\left(r_{i}+1\right)\left(x_{i}-\alpha\right)^{\beta}-n\left(x_{1}-\alpha\right)^{\beta}}{n(m-1)\left(x_{1}-\alpha\right)^{\beta}}
\end{aligned}
$$

hence, $T_{1}$ has an $F$-distribution with $2 m-2$ and 2 degrees of freedom. hence, for $0<\phi<1$, the event

$$
\begin{gathered}
1-\phi=P\left[F_{1-\frac{\phi}{2}}(2 m-2,2)<T_{1}<F_{\frac{\phi}{2}}(2 m-2,2)\right] \\
=P\left[\begin{array}{l}
F_{1-\frac{\phi}{2}}(2 m-2,2)<\frac{\sum_{i=1}^{m}\left(r_{i}+1\right)\left(x_{i}-\alpha\right)^{\beta}-n\left(x_{1}-\alpha\right)^{\beta}}{n(m-1)\left(x_{1}-\alpha\right)^{\beta}} \\
<F_{\frac{\phi}{2}}(2 m-2,2)
\end{array}\right] \\
=P\left[\begin{array}{l}
\frac{1}{m-1}+F_{1-\frac{\phi}{2}}(2 m-2,2)<\sum_{i=1}^{m}\left(\frac{r_{i}+1}{n(m-1)}\right)\left(\frac{X_{i}-\alpha}{X_{1}-\alpha}\right)^{\beta} \\
<\frac{1}{m-1}+F_{\frac{\phi}{2}}(2 m-2,2)
\end{array}\right]
\end{gathered}
$$

is equivalent to the event

$$
\begin{aligned}
\Psi\left(X_{1}, \cdots, X_{m},\right. & \left.F_{\left(1-\frac{\phi}{2}\right)(2 m-2,2)}\right)<\beta \\
& <\Psi\left(X_{1}, \cdots, X_{m}, F_{\left(\frac{\phi}{2}\right)(2 m-2,2)}\right) .
\end{aligned}
$$

Let us discuss the joint confidence region for the parameters $\beta$ and $\eta$. A naive $100(1-\phi) \%$ joint confidence may be constructed by using $B_{\phi}$ for $\left(T_{1}, T_{2}\right)$ such that

$$
B_{\phi}=\left\{\left(t_{1}, t_{2}\right) \mid g_{1}\left(t_{1}\right) g_{2}\left(t_{2}\right)>\beta_{\phi}\right\}, \quad 0<\phi<1
$$

where $g_{1}$ is the density function of $F$ distribution, $g_{2}$ is the density function of chi-square distribution, and $\beta_{\phi}$ is a constant given by

$$
\begin{aligned}
1-\phi & =\iint_{B_{\phi}} g_{1}\left(t_{1}\right) g_{2}\left(t_{2}\right) d t_{2} d t_{1} \\
& =\int_{l_{1}(\phi)}^{l_{2}(\phi)} g_{1}\left(t_{1}\right) \int_{\lambda\left(t_{1} \mid \phi\right)}^{\xi\left(t_{1} \mid \phi\right)} g_{2}\left(t_{2}\right) d t_{2} d t_{1} .
\end{aligned}
$$


Since $T_{1}$ and $T_{2}$ are independent, a simpler way is to choose $\xi$ and $\lambda$ depending only upon $\varphi$ such that

$$
\int_{l_{1}(\phi)}^{l_{2}(\phi)} g_{1}\left(t_{1}\right) d t_{1}=\sqrt{1-\phi}
$$

and

$$
\int_{\lambda(\phi)}^{\xi(\phi)} g_{2}\left(t_{2}\right) d t_{2}=\sqrt{1-\phi} .
$$

Let $\chi_{\phi(\delta)}^{2}$ denote the percentile of chi-square distribution with right-tail probability $\phi$ and $\delta$ degrees of freedom. An exact joint confidence region for the parameters $c$ and $b$ is given in the following theorem.

\section{Theorem 2.}

Suppose that $X_{i}, i=1, \cdots, m$, are the order statistics of a progressively type-II censored sample of size $n$ from a three parameter inverse Weibull distribution, with censoring scheme $\left(r_{1}, \cdots, r_{m}\right)$. Then a $100(1-\phi) \%$ joint confidence region for $\epsilon$ and $t$ is determined by the following inequalities:

$$
\left\{\begin{array}{l}
\Psi\left(X_{1}, \cdots, X_{m}, F_{\frac{1+\sqrt{1-\phi}}{2}(2 m-2,2)}\right)<\beta<\Psi\left(X_{1}, \cdots, X_{m}, F_{\frac{1-\sqrt{1-\phi}}{2}(2 m-2,2)}\right) \\
\frac{\chi_{\frac{1+\sqrt{1+\phi}}{2}}^{2}(2 m)}{\left[2 \sum_{i=1}^{m}\left(r_{i}+1\right)\left(X_{i}-\alpha\right)^{\beta}\right]^{\frac{1}{\beta}}}<\eta<\frac{\chi_{\frac{1+\sqrt{1-\phi}}{2}(2 m)}^{2}}{\left[2 \sum_{i=1}^{m}\left(r_{i}+1\right)\left(X_{i}-\alpha\right)^{\beta}\right]^{\frac{1}{\beta}}}
\end{array}\right.
$$

where $0<\phi<1$ and $\Psi\left(X_{1}, \cdots, X_{m}, t\right)$ is the solution of $\beta$ for the equation

$$
\frac{\sum_{i=1}^{m}\left(r_{i}+1\right)\left(x_{i}-\alpha\right)^{\beta}-n\left(x_{1}-\alpha\right)^{\beta}}{n(m-1)\left(x_{1}-\alpha\right)^{\beta}}=t
$$

Proof: From (1e10) and (1e11) we obtain

$$
\begin{aligned}
T_{1} & =\frac{\sum_{i=1}^{m}\left(r_{i}+1\right) Z_{i}-n Z_{1}}{n(m-1) Z_{1}} \\
& =\frac{\sum_{i=1}^{m}\left(r_{i}+1\right)\left(x_{i}-\alpha\right)^{\beta}-n\left(x_{1}-\alpha\right)^{\beta}}{n(m-1)\left(x_{1}-\alpha\right)^{\beta}}
\end{aligned}
$$

and

$$
\begin{aligned}
T_{2} & =2 \sum_{i=1}^{m}(+1) Y_{i} \\
& =2 \eta^{\beta} \sum_{i=1}^{m}\left(r_{i}+1\right)\left(x_{i}-\alpha\right)^{\beta}
\end{aligned}
$$

hence, $T_{1}$ has an $F$ distribution with $2 m-2$ and 2 degrees of freedom and $T_{2}$ has a chi-square distribution with $2 m$ degrees of freedom, $T_{1}$ and $T_{2}$ are independent. Then, for $0<\varphi<1$,

$$
\begin{aligned}
& 1-\phi=\sqrt{1-\phi} \sqrt{1-\phi} \\
& =P\left(F_{\frac{1+\sqrt{1-\phi}}{2}(2 m-2,2)}<T_{1}<F_{\frac{1-\sqrt{1-\phi}}{2}(2 m-2,2)}\right) \\
& \times P\left(\chi_{\frac{1+\sqrt{1-\phi}}{2}(2 m)}^{2}<T_{2}<\chi_{\frac{1-\sqrt{1-\phi}}{2}(2 m)}^{2}\right) \\
& =P\left(\begin{array}{l}
F_{\frac{1+\sqrt{1-\phi}}{2}(2 m-2,2)}<\frac{\sum_{i=1}^{m}\left(r_{i}+1\right)\left(x_{i}-\alpha\right)^{\beta}-n\left(x_{1}-\alpha\right)^{\beta}}{n(m-1)\left(x_{1}-\alpha\right)^{\beta}} \\
<F_{\frac{1-\sqrt{1-\phi}}{2}(2 m-2,2)}
\end{array}\right), \\
& \times P\left(\chi_{\frac{1+\sqrt{1-\phi}}{2}(2 m)}^{2}<2 \eta^{\beta} \sum_{i=1}^{m}\left(r_{i}+1\right)\left(x_{i}-\alpha\right)^{\beta}<\chi_{\frac{1-\sqrt{1-\phi}}{2}(2 m)}^{2}\right) \\
& =\Psi\left(X_{1}, \cdots, X_{m}, F_{\frac{1+\sqrt{1-\phi}}{2}(2 m-2,2)}\right)<\beta \\
& <\Psi\left(X_{1}, \cdots, X_{m}, F_{\frac{1-\sqrt{1-\phi}}{2}(2 m-2,2)}\right) \text {, } \\
& \frac{\chi_{\frac{1+\sqrt{1+\phi}}{2}(2 m)}^{2}}{\left[2 \sum_{i=1}^{m}\left(r_{i}+1\right)\left(X_{i}-\alpha\right)^{\beta}\right]^{\frac{1}{\beta}}}<\eta \\
& <\frac{\chi_{\frac{1+\sqrt{1-\phi}}{2}(2 m)}^{2}}{\left[2 \sum_{i=1}^{m}\left(r_{i}+1\right)\left(X_{i}-\alpha\right)^{\beta}\right]^{\frac{1}{\beta}}}
\end{aligned}
$$

\section{Illustrative Example}

To illustrate the use of the estimation methods discussed in the article, the following example is discussed.

Example. Consider a progressively type-II censored sample of size $m=8$ from a sample of size $n=20$ from the inverse Weibull distribution with $\beta=0.5, \eta=2$ and $\alpha=1$ was simulated, with censoring scheme $r=(0,0,3,0,4,0,0,5)$. The simulated progressively type-II censored sample is in this table.

\begin{tabular}{c|cccc}
\hline$i$ & 1 & 2 & 3 & 4 \\
$x_{i}$ & 1.7096 & 1.7401 & 2.3351 & 2.3352 \\
$r_{i}$ & 0 & 0 & 3 & 0 \\
$i$ & 5 & 6 & 7 & 8 \\
$x_{i}$ & 2.5429 & 3.2457 & 4.5363 & 9.4191 \\
$r_{i}$ & 4 & 0 & 0 & 5 \\
\hline
\end{tabular}

Using the iterative formula presented in section 2 , the $M L E S$ of $\beta$ and $\eta$ are $\hat{\beta}=0.9047$ and $\hat{\eta}=1.7665$, respectively. To find a $95 \%$ confidence interval for $\eta$, we need the percentiles 
$F_{0.025}(14,2)=39.4265$ and $F_{0.975}(14,2)=0.2059$.

By Theorem 1 and using the Mathematica nonlinear equation solver, the $95 \%$ confidence interval for $\beta$ is $(0.6053,2.7472)$. Furthermore, to obtain a $95 \%$ joint confidence region for $\beta$ and $\eta$, we need the percentiles $F_{0.0127}(14,2)=78.4147, F_{0.9873}(14,2)=0.1648$, $\chi_{0.0127}^{2}(16)=31.2069$ and $\chi_{0.9873}^{2}(16)=6.0684$ By Theorem 2 , a $95 \%$ joint confidence region for $\beta$ and $\eta$ is determined by the following inequalities:

$$
\left\{\begin{array}{l}
0.1118<\beta<3.0276 \\
\frac{6.0684}{2 \sum_{i=1}^{m}\left(r_{i}+1\right)\left(X_{i}-\alpha\right)^{-\beta}}<\eta<\frac{31.2069}{2 \sum_{i=1}^{m}\left(r_{i}+1\right)\left(X_{i}-\alpha\right)^{-\beta}} .
\end{array}\right.
$$

\section{Conclusion}

We use maximum likelihood method to obtain the point estimators of the parameters of three parameter inverse Weibull distribution based on progressive Type II censoring. We provide two pivotal quantities to construct an exact confidence interval and an exact joint confidence region for the parameters, respectively. A numerical data set is analyzed in section 4 to illustrate our approach.

\section{References}

[1] A. C. Cohen, Progressively censored samples in life testing. Techno metrics, 5, 327-339, (1963).

[2] N. R. Mann, Best linear invariant estimation for Weibull parameters under progressive censoring, Technometrics, 13, 521-533, (1971).

[3] J. Y. Wong, Simultaneously estimating the three Weibull parameters from progressively censored samples, Microelectronics and Reliability, 33, 2217- 2224, (1993).
[4] N. Balakrishnan, and R. Aggarwala, Progressive Censoring-Theory, Methods, and Applications, Birkhauser, Boston, SBN 978-0-8176-4001-9 e-book package (2000).

[5] Wu. Shuo-Jye Estimation of the parameters of the weibull distribution with progressively censored data, Journal of Japan Statistical Society, 2, 155-163, (2002).

[6] W. B. Nelson, Applied Life Data Analysis. John Wiley \& Sons, New York, (1982).

[7] R. Calaria, and G. Pulcini, On the maximum likelihood and least-squares estimation in the inverse Weibull distributions. Statistical Application, 2(1), 53-66, (1990).

[8] M. Maswedah, Conditional confidence interval estimation for the inverse Weibull distribution based on censored generalized order statistics. Journal of Statisticl Computation and Simulation, 73, 887-898, (2003).

[9] R. Dumonceaux, and C. E. Antle, Discrimination between the lognormal and Weibull distribution. Techno metrics, 15, 923-926, (1973).

[10] N. L. Johnson, S. Kotz, and N. Balakrishnan, Continuous Univariate Distributions. Vol. 2, second edition. John Wiley $\&$ Sons New York, (1995).

[11] D. N. P. Murthy, M. Xie, and R. Jiang, Weibull Model. John Wiley \& Sons, New York, (2004).

[12] M. M, Mohie El-Din,,and F. H. Riad, Estimation and Prediction for the Inverse Weibull Distribution Based on Records, Journal of Advanced Research in Statistics and Probability (JARSP), 3(2), 20 - 27, (2011).

[13] P. Erto, New Practical Bayes estimators for the 2-Parameter Weibull distribution, IEEE Transactions on Reliability R-31, 194-197, (1982),

[14] M. Marušić, , D. Marković, and D. Jukić , Least squares fitting the three-parameter inverse Weibull density, Math. Commun., Vol. 15, No. 2, pp. 539-553, (2010).

[15] D. R. Thomas, and W. M. Wilson, Linear order statistic estimation for the two parameter Weibull and extreme value distribution from type-II progressively censored samples, Technometrics, 14, 679-691, (1972). 